\title{
PROPUESTA INICIAL DE UNA RED AMPLIADA DE ÁREAS PROTEGIDAS EN ESPAÑA PENINSULAR BAJO EL ENFOQUE DE SU CONEXIÓN CON LA MATRIZ TERRITORIAL ${ }^{1}$
}

\author{
Javier Martínez-Vega² y Pilar Echavarría Daspet ${ }^{3}$ \\ Instituto de Economía, Geografía y Demografía \\ Centro de Ciencias Humanas y Sociales (CSIC)
}

\section{RESUMEN}

Se propone la oportunidad de ampliar la actual red de Áreas Protegidas de España peninsular bajo una estrategia global de conservación de la naturaleza que vaya más allá de la preservación de los espacios que ya disponen de una figura de protección. Mediante el empleo de Tecnologías de Información Geográfica se han diseñado zonas de amortiguación y corredores ecológicos para que funcionen como conectores de las áreas núcleo que son los actuales Espacios Naturales Protegidos. Esta red ecológica ampliada ocupa $345.733 \mathrm{~km}^{2}$, equivalente al $70 \%$ de la superficie total del área de estudio. A modo de validación, se ha combinado esta cobertura de Áreas Protegidas con mapas de rareza, representatividad y diversidad de los ecosistemas forestales. De acuerdo a estos indicadores de Ecología del Paisaje, el 82,25\% de los ecosistemas forestales más raros de España peninsular se encuentran dentro de la red ampliada de Áreas Protegidas propuesta, al igual que el $82,88 \%$ de los montes de mayor representatividad y el $89,21 \%$ de las áreas forestales más diversas.

Palabras clave: Redes ecológicas, Áreas Protegidas, zonas de amortiguación, corredores ecológicos, conectividad, TIG, España peninsular.

\section{ABSTRACT \\ A first proposal of an enlarged network of protected areas in the Iberian Peninsula (Spain), on the basis of their surrounding zones}

It offers the opportunity to expand the existing network of protected areas of mainland Spain under a global conservation strategy of nature that goes far beyond of the preservation of spaces that already have a protection figure. Through the use of Geographic Information Technologies are designed buffer zones and ecological corridors to function as connectors core areas are the current Protected Natural Areas. This extended ecological network occupies $345,733 \mathrm{~km}^{2}$, equivalent to the $70 \%$ of the total area of the study area. By way of validation, has combined this coverage maps Protected Areas scarcity, representativeness and diversity of forest ecosystems. According to these indicators of Landscape Ecology, $82.25 \%$ of scarce forest ecosystems mainland Spain are within the expanded network of protected areas proposed, like the $82.88 \%$ of the most representative mountains and $89.21 \%$ of forest areas more diverse.

Key words: Ecological networks, protected areas, buffer zones, ecological corridors, connectivity, TIG, mainland Spain.

\footnotetext{
Los resultados de este trabajo se encuentran vinculados a los proyectos FIREGLOBE (CGL2008-01083), financiado por el Ministerio de Ciencia e Innovación, y SUSTAINMED (FP7-KBBE-2009-3), financiado por el $7^{\circ}$ Programa Marco de la UE.

javier.martinez@cchs.csic.es

pilar.echavarria@cchs.csic.es
} 


\section{INTRODUCCIÓN}

La conservación de la naturaleza, la gestión de los recursos naturales y la pérdida de biodiversidad son algunas de las principales preocupaciones ambientales señaladas en la Estrategia Comunitaria de Biodiversidad, en la Estrategia Española para la Conservación y el Uso Sostenible de la Diversidad Biológica de 1998 (Ministerio de Medio Ambiente, 1999), en la Estrategia Española de Desarrollo Sostenible (Ministerio de Presidencia, 2007) y en los informes anuales del Observatorio de la Sostenibilidad en España (OSE, 2012). Las estrategias comunitaria y española recomiendan la necesidad de conservar más allá de los espacios protegidos e indican la importancia de los corredores ecológicos y de otras zonas no protegidas con alto valor ecológico y biodiversidad.

La Ley de Patrimonio Natural y de la Biodiversidad (Ley 42/2007) supone un cambio en la política española sobre diversidad biológica, al incorporar nuevos mecanismos para frenar las amenazas, limitando, especialmente, la expansión urbanística que pudiera afectar al medio natural y obligando a elaborar y respetar los planes de ordenación de recursos naturales. A pesar de este empeño, no siempre se han conseguido los objetivos perseguidos en el conjunto de la matriz territorial, ni siquiera en los espacios protegidos. La Estrategia Española de Desarrollo Sostenible reconoce la importancia de implantar acciones destinadas a preservar y restaurar la biodiversidad.

Otros instrumentos legales y de planificación sectorial se preocupan por incorporar la cuestión de la conservación de la naturaleza y de la biodiversidad en sus disposiciones de ámbito estatal o autonómico. Se repasan, a continuación, algunos que se consideran relevantes.

La Ley de Ordenación Agraria y Desarrollo Rural, que estudió y debatió el Gobierno de España en 2002 (Sancho et al., 2002), contemplaba una nueva realidad rural que dejaba atrás su primacía productiva para abrirse a una plurifuncionalidad y diversidad económica en la que las Áreas Protegidas, los servicios ambientales proporcionados por éstas y el turismo rural vinculado a ellas, jugaban un papel fundamental en el Desarrollo Rural.

El Programa de Desarrollo Rural Sostenible, contemplado en la Ley 45/2007, de 13 de diciembre, prevé, entre las múltiples medidas para el desarrollo rural sostenible, aquellas destinadas a la conservación de la naturaleza y a la gestión de los recursos naturales con el fin de preservar y mejorar la calidad del medio ambiente rural. Las áreas de la Red Natura 2000, los espacios protegidos, los hábitats de mayor interés y los municipios de escasa densidad demográfica y elevada significación de la actividad agraria son consideradas zonas rurales prioritarias a los efectos de la aplicación de este programa.

También, las leyes de Ordenación del Territorio tratan las cuestiones ambientales y la conservación de la naturaleza. Todas las leyes autonómicas, en esta materia, tienen una serie de rasgos comunes, entre los que es preciso destacar la necesidad de delimitar áreas de planificación ambiental o de especial protección por valores naturales y paisajísticos (EUROPARC-ESPAÑA, 2005).

De cualquier modo, la riqueza y singularidad de hábitats en España coloca a nuestro país en puestos relevantes, a nivel europeo y mundial. Internacionalmente, es preciso subrayar la importancia del Convenio de Naciones Unidas sobre Diversidad Biológica, mientras que, en Europa, es conocida la relevancia de las Directivas Aves (79/409/CEE) y Hábitats (92/43/CEE). La segunda recomienda, además, tener en cuenta los elementos del paisaje que dan coherencia a la Red Natura 2000, facilitando la conectividad entre las zonas núcleo. El Real Decreto 1997/1995, de 7 de diciembre, que traspone esta directiva al ordenamiento jurídico español, señala a las vías pecuarias como uno de los posibles conectores de los espacios protegidos.

Como ya es sabido, el diseño de redes ecológicas de ámbito internacional, nacional o autonómico es trascendental para preservar la diversidad biológica y conservar los hábitats y especies amenazados. En nuestro ámbito geográfico de interés, ése es el propósito de la Red Natura 2000 y de la red de Espacios Naturales Protegidos del estado español (de Lucio et al., 2008).

Así pues, es frecuentemente reconocida la insuficiencia de las redes ecológicas para alcanzar los objetivos de conservación de la diversidad biológica y la importancia de complementarlas, incorporando nuevas áreas de interés natural e interconectando las áreas-núcleo entre sí mediante corredores biológicos que favorezcan el movimiento, dispersión e intercambio de las especies protegidas y de sus poblaciones. 
Éste es uno de los principales retos señalados en el Plan de Acción para los Espacios Naturales Protegidos del estado español: "desarrollar sistemas de áreas protegidas que integren todos los elementos necesarios para garantizar la diversidad biológica y paisajística del territorio (áreas núcleo, zonas de amortiguación, corredores)" (EUROPARC-ESPAÑA, 2002, p. 13).

Múltiples son los trabajos que inciden en esta línea. Uno de los principales es el publicado por Bennett y Mulongoy (2006). En él, se repasan los principales conceptos y se hace una revisión de experiencias con redes ecológicas, corredores y zonas de protección. Esta revisión responde a la necesidad identificada por el Grupo de Trabajo en Áreas Protegidas, en relación con la Decisión VII/28, adoptada en la Seventh Conference of the Parties to the Convention on Biological Diversity, celebrada en Kuala Lumpur, en 2004.

Investigaciones previas sobre redes y corredores ecológicos han centrado su atención sobre una gran variedad de espacios geográficos (Walker y Craighead, 1997; Laurance y Laurance, 1999; Silori y Mishra, 2001; Woess et al., 2002; Jordán et al., 2003; Tubelis et al., 2004; Wissmar, 2004; Brown y Harris, 2005; Bullock y Samways, 2005; Weber et al., 2006; Graves et al., 2007; Nandy et al., 2007, Roe y Georges, 2007; Vogt et al., 2007; Kaligaric et al., 2008; Parker et al., 2008, Roy y Blois, 2008).

Destacan algunas investigaciones focalizadas en el estudio de corredores riparios y fluviales (Wissmar, 2004; Kaligaric et al., 2008; Tormos et al., 2011; Clerici y Vogt, 2013), el examen de la permeabilidad de las infraestructuras viarias y su influencia sobre la pérdida de conectividad de los hábitats de la Red Natura 2000 (Martín, et al., 2008; Gurrutxaga et al., 2011), en la conectividad del paisaje para la vida silvestre en el proceso de planificación de nuevas carreteras (Woess et al., 2002), en el análisis del grado de cobertura y protección de los bosques a nivel regional (Gurrutxaga, 2008), en la conexión de hábitats en paisajes humanizados y urbanizados mediante corredores ecológicos (Parker et al., 2008; Gurrutxaga et al., 2010), en la identificación y priorización de áreas de mayor importancia ecológica (Weber et al., 2006), en la cooperación transfronteriza entre países involucrados en la conservación de una misma red ecológica internacional (Leibenath et al., 2010) y en el papel de los actores y tomadores de decisiones involucrados (Suskevics et al., 2013).

Los métodos más empleados para alcanzar estos propósitos han sido los análisis cluster (Arendt, 2004; Graves et al., 2007), las técnicas de regresión múltiple (Laurance y Laurance, 1999; Roy y Blois, 2008), los modelos lineales mixtos generalizados (Tubelis et al., 2004), modelos de simulación espacialmente explícita (Jepsen et al., 2005), modelos conceptuales de conectividad ecológica (Parker et al., 2008), técnicas y modelos de ayuda a la decisión (Hilty et al., 2006), análisis GAP (Walker y Craighead, 1997; Gurrutxaga, 2008), dimensión fractal (Kaligaric et al., 2008), encuestas a propietarios (Brown y Harris, 2005), la cartografía automática de corredores a partir del procesado morfológico de imágenes de satélite (Vogt et al., 2007), el análisis multitemporal de la matriz de cambios con imágenes de satélite mediante análisis visual (Nandy et al., 2007), con imágenes de alta resolución espacial (Tormos et al., 2011), técnicas de segmentación y análisis multiescalar (Clerici y Vogt, 2013) y un enfoque o método ecosistémico (Bennett y Mulongoy, 2006), articulado como un esquema para la acción y para una toma de decisiones de carácter holístico.

En España, se han presentado diversas experiencias en esta línea, en un taller sobre conectividad ecológica (EUROPARC-ESPAÑA, 2008). Una de ellas está relacionada con la coherencia global de la Red Natura 2000 en España. Otra tiene por objetivo diseñar una red de corredores ecológicos que asegure la coherencia de la Red Natura 2000 en la Región de Murcia. La última estudia la conectividad ecológica en la provincia de Barcelona desde una concepción holística. Como fruto del intercambio de experiencias se ha propuesto la creación de un grupo de trabajo sobre conectividad ecológica y la recopilación de proyectos y experiencias sobre este tema.

Por último, recientes trabajos e iniciativas inciden en la necesidad de realizar una evaluación de la eficacia de las Áreas Protegidas. No basta con declarar un conjunto de Áreas Protegidas sino que es necesario conservar, planificar y gestionar, adecuadamente, sus recursos naturales, involucrar a la población residente y visitante en la custodia de sus territorios y recursos naturales y disponer de los recursos humanos y financieros necesarios para asegurar una gestión adecuada. Recientemente, CSIC y la Fundación BBVA ha organizado el I Taller Nacional sobre Evaluación de la Eficacia de las Áreas Protegidas, dando a conocer las publicaciones de Rodríguez-Rodríguez y Martínez-Vega (2012, 2013a, 2013b) 
y debatiendo sobre sus contenidos. En este taller han participado gestores nacionales y autonómicos, responsables de Áreas Protegidas, así como representantes de organizaciones especializadas en este tema, de asociaciones ecologistas e investigadores. Se discutió acerca de la pertinencia de un sistema de evaluación estandarizado para España y de los indicadores que formarían parte de este sistema de evaluación. Entre otros muchos, destaca ahora la conveniencia de incluir una serie de indicadores que midan la fragmentación, el aislamiento y la accesibilidad de las Áreas Protegidas, considerados éstos como indicadores de presión y amenazas que restarían puntuación al índice global de eficacia. En todos los casos, un análisis espacial de la matriz territorial circundante a las Áreas Protegidas es fundamental.

\section{OBJETIVO}

El objetivo principal de este trabajo es proponer una metodología operativa, apoyada en herramientas SIG, para diseñar una red de espacios de interés natural, en el territorio peninsular español, interconectados geográficamente entre sí y complementarios de los que ya se encuentran protegidos por las redes autonómica, estatal y europea. El fin de esta red ampliada es extender la conservación de la naturaleza, mediante la incorporación de dos figuras adicionales a las zonas ya reguladas. Por un lado, las zonas de amortiguación que rodean y protegen a los espacios naturales de mayor valor, absorbiendo los impactos ambientales que, eventualmente, pudieran afectarlos. Por otro, añadiendo los corredores biológicos, de tipo fluvial, que facilitan la conectividad entre los espacios de mayor valor. Se pretende que esta red ampliada de Áreas Protegidas del ámbito peninsular español, inserta en su matriz territorial, sea la base que facilite, posteriormente, la regulación, ordenación y planificación de los usos del suelo bajo un enfoque dinámico de mayores restricciones en las zonas núcleo hasta menores en las zonas exteriores de influencia. En otras palabras, se persigue su sostenibilidad. Los eventuales Planes de Ordenación de Recursos Naturales (PORN) y los Planes Rectores de Uso y Gestión (PRUG) de estas nuevas figuras complementarias de protección podrían ser tenidos en cuenta en la simulación de futuros escenarios verdes de estos territorios mediante modeladores basados en técnicas de regresión logística (CLUE) y de redes neuronales (LCM-IDRISI) (Gallardo y Martínez-Vega, 2012).

El interés de las poblaciones locales por sus Áreas Protegidas justifica, de alguna forma, este objetivo a pesar del contexto de crisis económica en el que estamos envueltos y de las limitaciones de actividades que imponen la planificación y gestión de estas zonas (Rodríguez-Rodríguez, 2012). Cada día existe mejor disposición al pago por el uso y visita de estas áreas y ellas proporcionan mayores servicios ambientales que son más apreciados por las poblaciones residentes y visitantes.

\section{3. ÁREA DE ESTUDIO}

Como se ha comentado anteriormente, este trabajo exploratorio deriva de la información generada en el marco del proyecto FIREGLOBE (Chuvieco et al., 2012; Martínez-Vega y Chuvieco, 2012). Aunque este proyecto ha centrado su atención en dos escalas -global y nacional- en este trabajo nos centraremos en la escala nacional, aunque el proyecto se ha focalizado, exclusivamente, en el territorio de España peninsular. Así pues, se han dejado fuera las tres provincias de los archipiélagos balear y canario.

En conjunto, España peninsular abarca un territorio compuesto por 47 provincias que ocupan una superficie total de $493.715 \mathrm{~km}^{2}$, poblada por 43.862 .718 hab., según el último censo de 2012, con una densidad promedio ligeramente inferior a $89 \mathrm{hab} / \mathrm{km}^{2}$. Según los datos de CORINE Land Cover 2006, las áreas forestales ocupan una superficie de $254.058 \mathrm{~km}^{2}$ que equivale al $51,5 \%$ de la superficie total. En el área de estudio hay presencia de espacios forestales bien conservados, de alto valor ecológico y paisajístico, que contienen endemismos y zonas con altos índices de rareza y singularidad en el conjunto de la UE.

Asimismo, contiene numerosas Áreas Protegidas, bajo distintas figuras de protección reguladas por distintos mecanismos e integradas en distintas redes de protección. Según los datos de EUROPARC-España (2012), en España peninsular el 12,4\% de su superficie terrestre se encuentra protegida, lo que equivale a 6.101.048 ha. Adicionalmente, si consideramos los espacios terrestres incluidos en Red Natura 2000, hay que reseñar que España es el país de la UE que más superficie terrestre 
aporta a esta red ecológica continental. Tan sólo el territorio de España peninsular incorpora un total de 13.650.676 ha, lo que supone el $27,7 \%$ de la superficie terrestre de la red. En este territorio están representadas 3 de las 9 regiones biogeográficas de Europa. No obstante, conviene considerar que existe solapamiento geográfico entre las figuras de protección.

También hay abundancia de otras áreas de interés natural que, sin pertenecer ahora a ninguna red de espacios protegidos, pueden complementar la disponibilidad de recursos naturales, susceptibles de ser regulados o protegidos en un futuro.

Sin embargo, las principales amenazas para su conservación son la elevada presión demográfica, recreativa y urbanística de algunos ecosistemas naturales, especialmente los más próximos a las grandes urbes. Los ecosistemas forestales se encuentran amenazados por el inicio de incendios forestales como consecuencia de las variadas negligencias y por la rápida propagación de los incendios forestales debido a las características del territorio, de las condiciones físicas y de una insuficiente gestión forestal (selvicultura preventiva, limpieza de las franjas de seguridad, etc.). Frecuentemente, los incendios afectan a lugares de elevado valor ecológico y a espacios de la Red Natura 2000.

Por otro lado, las áreas protegidas se ven amenazadas por la fragmentación de las numerosas teselas de la matriz territorial en las que se encuentran insertas y por su aislamiento y pérdida de conectividad con otras áreas protegidas vecinas como consecuencia de la construcción de grandes infraestructuras viarias (autopistas, autovías, infraestructuras ferroviarias de Alta Velocidad), aeroportuarias y portuarias. Por otra parte, en las áreas forestales se encuentran montes que han perdido su funcionalidad tradicional y se encuentran abandonados, sin ser gestionados adecuadamente. Por último, puede decirse que existe ausencia de una planificación del territorio no urbano y de la ordenación de los recursos forestales. En diversos lugares se constata la pérdida de suelo por los usos inadecuados en zonas sensibles donde el riesgo de erosión es moderado o elevado.

\section{MATERIAL Y MÉTODOS}

Los datos utilizados como fuentes de información de este trabajo han sido diversos. Con objeto de localizar lo que llamaremos las áreas núcleo se ha compilado la siguiente información cartográfica procedente, la mayor parte de ella, del Banco de Datos de la Biodiversidad del Ministerio de Agricultura, Alimentación y Medio Ambiente que, a su vez, recoge y coordina información geográfica producida o suministrada por las Comunidades Autónomas, universidades y por organizaciones de conservación de la naturaleza:

- Cobertura de Espacios Naturales Protegidos que incluye diversas figuras de protección: Parques Nacionales, Parques Naturales, Otros Parques (Regionales, Rurales, etc.), Reservas Naturales, Otras Reservas, Paisajes Protegidos, Parajes, Monumentos Naturales, Biotopos Protegidos y Otras Figuras).

- Espacios de la Red Natura 2000: Zonas de Especial Protección para las Aves y Lugares de Importancia Comunitaria.

- Áreas Importantes para las Aves (IBAS), designadas por la Sociedad Española de Ornitología.

- Reservas de la Biosfera del Programa MAB de la UNESCO.

- Humedales de importancia internacional inscritos en la lista del Convenio RAMSAR.

- Zonas Especialmente Protegidas de Importancia para el mar Mediterráneo (ZEPIM).

Dada la gran variedad de figuras de protección existentes y con el objetivo de poder comparar los resultados con los de otras zonas geográficas del mundo, se consideró pertinente realizar una armonización de las áreas protegidas peninsulares para equipararlas con las categorías oficiales de la Unión Internacional de Conservación de la Naturaleza (tabla 1). 
Tabla 1. Equivalencias entre las figuras regionales, nacionales e internacionales de protección de las Áreas Protegidas.

\begin{tabular}{|c|c|c|}
\hline Categorías a nivel de Comunidades Autónomas & $\begin{array}{l}\text { Grupos de Categorías } \\
\text { para España (incluyendo } \\
\text { categorías internacionales) }\end{array}$ & Categoría UICN \\
\hline $\begin{array}{l}\text { Reserva Natural, Reserva Natural Concertada, Reserva Natural } \\
\text { Dirigida, Reserva Natural Especial, Reserva Natural Integral, } \\
\text { Reserva Natural Parcial, Reserva, Reserva Integral, Reserva Ley } \\
\text { de Protección Propia, Sitio de Interés Científico, Sitio Natural de } \\
\text { Interés Nacional }\end{array}$ & Reserva Natural & $\begin{array}{l}\text { I. Strict Natural Reserve /Wild } \\
\text { Natural Area }\end{array}$ \\
\hline Parque Nacional & Parque Nacional & II. National Park \\
\hline $\begin{array}{l}\text { Monumento Natural, Monumento Natural de Interés Nacional, } \\
\text { Árbol Singular, Enclave Natural, Geoparque, Área Natural Singular }\end{array}$ & $\begin{array}{l}\text { Monumento Natural, } \\
\text { Geoparque }\end{array}$ & III. Natural Monument \\
\hline $\begin{array}{l}\text { Reserva Natural de Fauna Salvaje, Reserva de Fauna, Reserva } \\
\text { Fluvial, Refugio de Fauna, Reserva Biogenética, Régimen de } \\
\text { Protección Preventiva, Biotopo Protegido, Corredor Ecológico y } \\
\text { de Biodiversidad, Humedal Protegido, Humedal de Importancia } \\
\text { Internacional (Ramsar), microrreserva. }\end{array}$ & $\begin{array}{l}\text { Reserva Natural, Reserva } \\
\text { Biogenética, Humedal de } \\
\text { Importancia internacional } \\
\text { (Ramsar) }\end{array}$ & $\begin{array}{l}\text { IV. Areas of Habitats and } \\
\text { Species Management }\end{array}$ \\
\hline $\begin{array}{l}\text { Paisaje Protegido, Paraje Pintoresco, Paraje Natural Municipal, } \\
\text { Paraje Natural, Paraje Natural de Interés Nacional, Parque } \\
\text { Periurbano, Parque Periurbano de Conservación y Ocio, Área } \\
\text { Natural Recreativa }\end{array}$ & Paisaje Protegido & $\begin{array}{l}\text { V. Protected Land or Sea } \\
\text { Landscape }\end{array}$ \\
\hline $\begin{array}{l}\text { Parque Natural, Parque Regional, Parque Rural, Plan Especial } \\
\text { de Protección, Lugar de Interés Comunitario, Zona de Especial } \\
\text { Protección para las Aves, Zona Especial de Conservación, Zona } \\
\text { de Especial Conservación de Importancia Comunitaria, Zona } \\
\text { de Especial Protección de los Valores Naturales, Zona de Interés } \\
\text { Regional, Zona de la Red Ecológica Europea Natura 2000, Zona } \\
\text { de Especial Conservación, Plan de Espacios de Interés Natural de } \\
\text { Cataluña (PEIN), Zona Especialmente Protegida de Importancia } \\
\text { para el Mediterráneo (ZEPIM), Espacio Natural Protegido }\end{array}$ & $\begin{array}{l}\text { Parque Natural, Reserva } \\
\text { de la Biosfera, Espacio } \\
\text { Protegido Red Natura } \\
\text { 2000, Zona Especialmente } \\
\text { Protegida de Importancia } \\
\text { para el Mediterráneo } \\
\text { (ZEPIM) }\end{array}$ & $\begin{array}{l}\text { VI. Protected area with } \\
\text { managed resources. }\end{array}$ \\
\hline
\end{tabular}

Fuente: EUROPARC-España, 2005b.

De la planimetría de la base cartográfica digital, EUROPA ESRI, actualizada en 2008, que forma parte de la colección de datos llamada ESRI Data \& Maps, se han extraído los ríos permanentes y láminas de agua con objeto de poder generar los corredores ecológicos de tipo fluvial. Se han excluido las corrientes de agua artificiales como los canales y otras infraestructuras de riego.

Del proyecto europeo SUSTAINMED se ha utilizado la cobertura de polígonos NUTS3, equivalentes a las provincias españolas, con objeto de calcular la superficie protegida actual, la superficie de la red ampliada de Áreas Protegidas y poder comparar así las diferencias entre ambas opciones a este nivel de detalle. Se han disuelto las fronteras interiores, conservando el perímetro exterior de las provincias litorales y de las provincias fronterizas con Portugal y Francia a modo de perímetro de España peninsular.

Se ha utilizado el mapa de CORINE-Land Cover 2006 para reclasificar las clases de usos del suelo en tres grandes dominios: forestal, agrícola y urbano.

Asimismo, se han utilizado los mapas de rareza, representatividad y diversidad del proyecto FIREGLOBE (Martínez Vega et al., 2012), con objeto de conocer qué proporción de los territorios de mayor valor ecológico están contenidos en la red ampliada de Áreas Protegidas propuesta en este trabajo.

La metodología se basa en el enfoque aportado por Bennett y Mulongoy (2006), en el contexto de la revisión de experiencias con redes ecológicas, corredores y zonas de amortiguación con objeto de aportar ideas para integrar las Áreas Protegidas en redes más amplias que mantengan la estructura y funcionalidad ecológica y las relaciones con Áreas Protegidas vecinas.

Se han utilizado las funciones básicas de análisis espacial, en un SIG. Mediante la herramienta de diseño de buffers, se ha dibujado un perímetro de protección de $5.000 \mathrm{~m}$. alrededor de los Espacios Naturales Protegidos y de los espacios de la Red Natura 2000 terrestres, a modo de zonas de amortiguación. No se 
han considerado las áreas protegidas marinas. Asimismo, alrededor de los cursos de agua permanentes y de las láminas de agua se han señalado corredores fluviales. En función de su contexto geográfico, se les ha dado un tratamiento desigual. Cuando los cursos o láminas de agua atraviesan zonas de vegetación silvestre y seminatural o cuando estas zonas se encuentran a menos de $10 \mathrm{~m}$. de distancia de aquellos, el corredor fluvial tiene un ancho de $750 \mathrm{~m}$, a cada lado de la entidad hidrográfica. Sin embargo, cuando las aguas atraviesan zonas de cultivos, estos corredores biológicos se han estrechado hasta $150 \mathrm{~m}$., a cada lado. Los buffers de las zonas de amortiguación y de los corredores ecológicos terrestres han sido cortados con el perímetro de España peninsular, excluyendo las zonas de influencia incluidas en espacios marinos.

Como después se verá en los resultados, mediante el diseño de las zonas de amortiguación y de los corredores ecológicos, las áreas núcleo quedan conectadas todas entre sí, evitando su aislamiento y reduciendo la fragmentación de la matriz territorial que queda entre las áreas núcleo.

Por último, alrededor de las zonas urbanas se ha dibujado un buffer de $1.000 \mathrm{~m}$., con objeto de excluir, de esta propuesta de red ampliada de Áreas Protegidas, estas zonas periurbanas en proceso de cambio, que reciben mayores influencias y presiones antrópicas y que fragmentan las Áreas Protegidas, dificultando la conectividad entre unas zonas núcleo y otras. También han sido eliminados los buffer de las zonas urbanas, localizados fuera del perímetro administrativo de España peninsular.

\section{RESULTADOS}

La red ampliada de Áreas Protegidas propuesta, compuesta por los Espacios Naturales Protegidos y por los sitios de la Red Natura 2000, por sus zonas de amortiguación y por los corredores fluviales, ocupa una superficie de $345.733 \mathrm{~km}^{2}$, lo que significa un $70 \%$ del territorio peninsular español.

En la figura 1 se observa la distribución espacial del conjunto de la red ampliada propuesta sobre el territorio peninsular español. En ella, se pueden diferenciar claramente los espacios protegidos actuales o áreas núcleo (en verde oscuro), las zonas de amortiguación que rodean a las primeras y los corredores ecológicos (en verde claro) ${ }^{4}$ que conectan las áreas núcleo y las zonas de amortiguación entre sí, siguiendo los cuerpos de agua y los cursos fluviales naturales permanentes, evitando, en todo momento, las actuales áreas urbanas y sus zonas de influencia inmediata.

Figura 1. Mapa de la red ampliada propuesta de Áreas Protegidas en España peninsular.

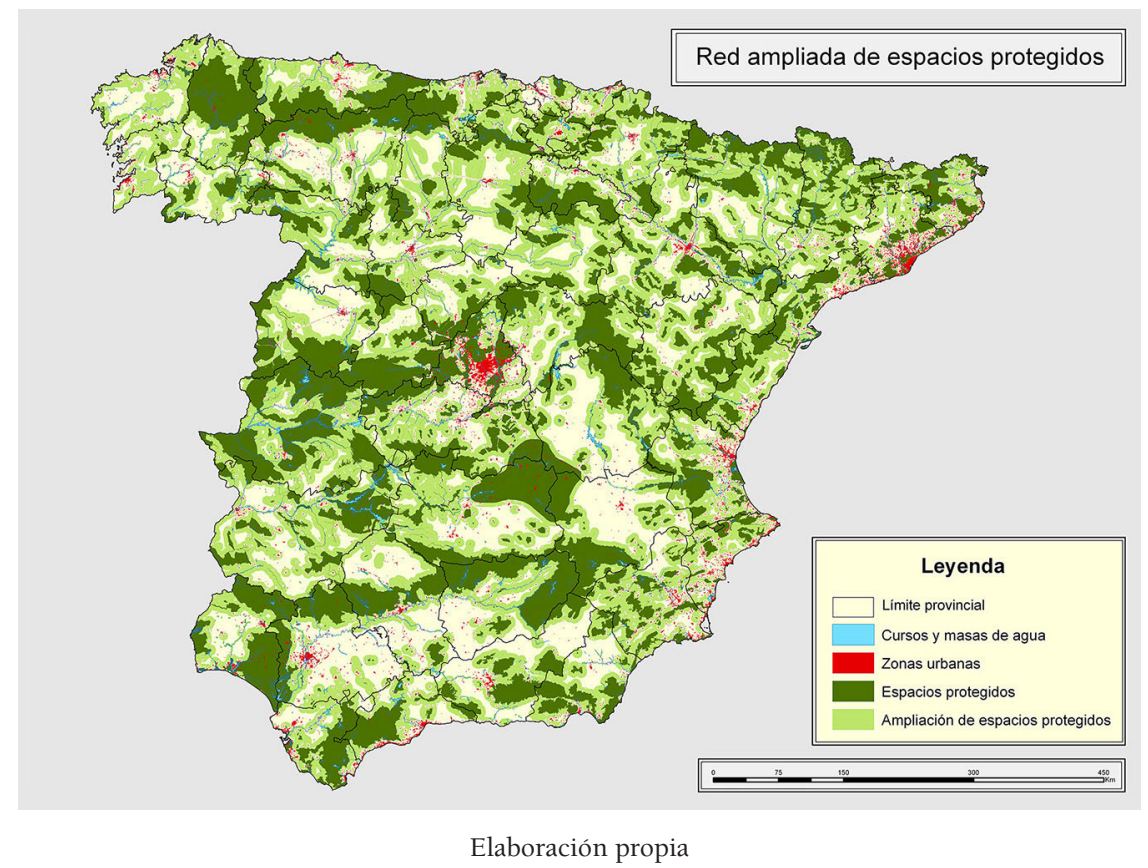

4 En esta figura no se han diferenciado las zonas de amortiguación y los corredores ecológicos para no dificultar su lectura cartográfica. 
Como se puede apreciar, existe una conectividad aceptable entre las Áreas Protegidas actuales -áreas núcleo- situadas en áreas de montaña, especialmente siguiendo la cornisa cantábrica y Pirineos. Al sur de la depresión del Ebro, existe cierto encadenamiento entre los Picos de Urbión, la sierra de la Demanda y la Ibérica. Igualmente, las áreas núcleo ocupan buena parte del Sistema Central y de la Serranía de Cuenca, de Sierra Morena y Doñana y de Sierra Nevada hasta la Serranía de Ronda. En la Comunidad de Madrid y en Cataluña, las áreas metropolitanas de Madrid y Barcelona fragmentan la matriz territorial y son las responsables de la interrupción geográfica de los espacios protegidos.

Por el contrario, en las dos mesetas y en las dos grandes depresiones -Ebro y Guadalquivir- existe cierta desconexión entre unas áreas núcleo y sus vecinas como consecuencia de la inmensidad geográfica de estas grandes unidades fisiográficas. En estos casos, es muy evidente, y elocuente gráficamente, el papel de conectores que juegan las áreas de amortiguación y los corredores ecológicos que siguen las riberas de los principales ríos -Duero, Tajo, Guadiana, Guadalquivir y Ebro- y de sus afluentes.

De acuerdo con Bennett y Mulongoy (2006), las zonas de amortiguación deben proteger al conjunto de las áreas núcleo de influencias externas potencialmente dañinas y deben ser consideradas áreas esencialmente de transición, caracterizadas por usos compatibles y sostenibles.

Los corredores biológicos, en el sentido de uniones funcionales entre sitios de interés ecológico, son dispositivos que mantienen un grado de coherencia entre estos ecosistemas, con objeto de paliar su fragmentación (Bennett y Mulongoy, 2006). También, se dice que son espacios que tienen, usualmente, una forma alargada y que facilitan la movilidad de los organismos entre distintos hábitats que están conectados entre sí a través de estos corredores, pudiendo ser analizados a distintos niveles y escalas, desde la intercontinental hasta la local (Hilty et al., 2006).

Esta propuesta ampliada supone un incremento de $187.582 \mathrm{~km}^{2}$ adicionales a la red actual de Áreas Protegidas, lo que significa casi duplicar la superficie actual (tabla 2). Por otra parte la red propuesta gana en conectividad a la red actual. Tras un análisis de las 479 manchas mayores de 3 ha que forman parte de esta red ampliada, tan sólo 6 de ellas, mayores de 50.000 ha cada una, ocupan el 98,06\% de la superficie total de la red, existiendo grandes y múltiples corredores que conectan unas grandes manchas con otras.

Tabla 2. Superficie de las Áreas Protegidas propuestas.

\begin{tabular}{|c|c|c|c|c|c|c|}
\hline NUTS & Provincias & $\begin{array}{c}\text { Superficie } \\
\text { geográfica } \\
\left(\mathbf{k m}^{2}\right)\end{array}$ & $\begin{array}{c}\text { AP actuales } \\
\left(\mathbf{k m}^{2}\right)\end{array}$ & $\begin{array}{c}\text { AP actuales } \\
(\%)\end{array}$ & $\begin{array}{c}\text { AP ampliadas } \\
\left(\mathbf{k m}^{2}\right)\end{array}$ & $\begin{array}{c}\text { AP ampliadas } \\
(\%)\end{array}$ \\
\hline ES111 & A Coruna & 7.983 & 436 & 5,46 & 4.475 & 56,05 \\
\hline ES112 & Lugo & 9.878 & 5.985 & 60,59 & 8.467 & 85,71 \\
\hline ES113 & Ourense & 7.294 & 1.460 & 20,01 & 4.382 & 60,08 \\
\hline ES114 & Pontevedra & 4.511 & 255 & 5,66 & 2.842 & 63,01 \\
\hline ES120 & Asturias & 10.610 & 4.288 & 40,42 & 8.138 & 76,70 \\
\hline ES130 & Cantabria & 5.317 & 1.913 & 35,98 & 4.097 & 77,07 \\
\hline ES211 & Álava & 3.035 & 884 & 29,12 & 2.523 & 83,14 \\
\hline ES212 & Guipúzcoa & 1.979 & 428 & 21,63 & 1.261 & 63,71 \\
\hline ES213 & Vizcaya & 2.215 & 425 & 19,21 & 1.261 & 56,94 \\
\hline ES220 & Navarra & 10.386 & 2.691 & 25,91 & 7.949 & 76,54 \\
\hline ES230 & La Rioja & 5.042 & 2.581 & 51,19 & 3.981 & 78,95 \\
\hline ES241 & Huesca & 15.647 & 5.077 & 32,45 & 12.550 & 80,20 \\
\hline ES242 & Teruel & 14.810 & 4.358 & 29,42 & 11.149 & 75,28 \\
\hline ES243 & Zaragoza & 17.273 & 4.320 & 25,01 & 11.988 & 69,40 \\
\hline ES300 & Madrid & 8.023 & 3.523 & 43,91 & 3.916 & 48,82 \\
\hline
\end{tabular}




\begin{tabular}{|c|c|c|c|c|c|c|}
\hline ES411 & Ávila & 8.049 & 4.407 & 54,75 & 6.710 & 83,37 \\
\hline ES412 & Burgos & 14.282 & 3.211 & 22,49 & 10.265 & 71,87 \\
\hline ES413 & León & 15.590 & 5.449 & 34,95 & 10.072 & 64,61 \\
\hline ES414 & Palencia & 8.049 & 1.872 & 23,25 & 5.944 & 73,85 \\
\hline ES415 & Salamanca & 12.361 & 4.387 & 35,49 & 8.064 & 65,24 \\
\hline ES416 & Segovia & 6.918 & 1.795 & 25,94 & 4.525 & 65,41 \\
\hline ES417 & Soria & 10.299 & 2.404 & 23,35 & 7.844 & 76,16 \\
\hline ES418 & Valladolid & 8.109 & 1.747 & 21,54 & 5.416 & 66,80 \\
\hline ES419 & Zamora & 10.569 & 3.238 & 30,63 & 8.134 & 76,96 \\
\hline ES421 & Albacete & 14.920 & 4.744 & 31,80 & 8.824 & 59,14 \\
\hline ES422 & Ciudad Real & 19.801 & 7.301 & 36,87 & 14.674 & 74,11 \\
\hline ES423 & Cuenca & 17.130 & 4.273 & 24,94 & 9.964 & 58,17 \\
\hline ES424 & Guadalajara & 12.203 & 4.419 & 36,21 & 9.396 & 77,00 \\
\hline ES425 & Toledo & 15.363 & 5.080 & 33,07 & 11.195 & 72,87 \\
\hline ES431 & Badajoz & 21.791 & 5.683 & 26,08 & 15.904 & 72,98 \\
\hline ES432 & Cáceres & 19.889 & 10.125 & 50,91 & 17.748 & 89,24 \\
\hline ES511 & Barcelona & 7.755 & 1.930 & 24,88 & 3.996 & 51,52 \\
\hline ES512 & Girona & 5.937 & 2.372 & 39,94 & 4.274 & 71,99 \\
\hline ES513 & Lleida & 12.196 & 4.106 & 33,67 & 10.372 & 85,04 \\
\hline ES514 & Tarragona & 6.315 & 2.200 & 34,83 & 4.693 & 74,32 \\
\hline ES521 & Alicante & 5.819 & 1.261 & 21,67 & 3.258 & 55,99 \\
\hline ES522 & Castellón & 6.638 & 2.340 & 35,25 & 5.333 & 80,33 \\
\hline ES523 & Valencia & 10.808 & 3.350 & 31,00 & 7.429 & 68,73 \\
\hline ES611 & Almería & 8.769 & 2.802 & 31,95 & 5.884 & 67,10 \\
\hline ES612 & Cádiz & 7.443 & 2.734 & 36,73 & 5.277 & 70,90 \\
\hline ES613 & Córdoba & 13.770 & 3.490 & 25,34 & 7.716 & 56,04 \\
\hline ES614 & Granada & 12.639 & 3.326 & 26,32 & 7.025 & 55,58 \\
\hline ES615 & Huelva & 10.149 & 5.381 & 53,02 & 7.503 & 73,93 \\
\hline ES616 & Jaén & 13.487 & 5.640 & 41,82 & 9.405 & 69,74 \\
\hline ES617 & Málaga & 7.308 & 2.497 & 34,17 & 4.911 & 67,20 \\
\hline ES618 & Sevilla & 14.045 & 3.273 & 23,30 & 7.151 & 50,91 \\
\hline \multirow[t]{2}{*}{ ES620 } & Murcia & 11.310 & 2.693 & 23,81 & 7.845 & 69,36 \\
\hline & & 493.715 & 158.151 & 32,03 & 345.733 & 70,03 \\
\hline
\end{tabular}

Fuente: SIG. Elaboración propia.

A nivel administrativo hay grandes diferencias entre unas provincias y otras. Los casos más extremos son Coruña y Pontevedra que multiplican por más de 10 veces la superficie protegida en esta red ampliada respecto a la existente. Ello se debe al gran número de cuerpos y corrientes de agua permanentes, a las extensas zonas forestales y a la escasa superficie urbana y agrícola. El caso contrario es Madrid. Se trata de un caso particular muy llamativo. A pesar de la extensa superficie urbana y artificial y al continuado proceso de crecimiento urbano de la región a lo largo de las últimas décadas, la Comunidad de Madrid protege, en la actualidad, casi un $44 \%$ de su superficie geográfica mediante la designación de 10 Espacios Naturales Protegidos y de un amplio número de zonas de la Red Natura 2000. En este trabajo, se propone la protección de otros $400 \mathrm{~km}^{2}$, apenas un 5\% más del territorio regional. Ello se debe a que se excluyen de esta propuesta las áreas urbanas y sus zonas de influencia correspondientes que, en esta región, son muy extensas. 
En toda la red ampliada -áreas núcleo, zonas de amortiguación y corredores ecológicos-, los gestores del territorio deben prestar especial atención a la regulación de usos del suelo para desterrar aquellos que puedan ser considerados poco sostenibles. Para facilitar la gestión y una alerta temprana a los gestores de estos espacios, sería muy conveniente hacer un seguimiento de las tendencias y evolución de los usos del suelo (CORINE-Land Cover 1990-2000-2006 y el futuro mapa de 2012 o mapas de SIOSE) y cruzar estas coberturas con un mapa de usos potenciales o de capacidad agroforestal elaborado mediante el sistema FAO (1976) o mediante sistemas expertos como MicroLEIS (de la Rosa et al., 2004, 2009). De esta manera, los expertos pueden realizar un seguimiento exhaustivo y localizar, de forma temprana, posibles incompatibilidades entre los usos del suelo y la capacidad de uso de las tierras, poniendo los medios necesarios para reconducir posibles amenazas y usos incompatibles con los objetivos de conservación.

\section{APROXIMACIÓN A UNA VALIDACIÓN}

A falta de una posibilidad real de validación de los resultados y de una consulta a expertos, que se realizará próximamente, se ha probado a comparar los resultados obtenidos en esta propuesta de red ampliada de Áreas Protegidas con los mapas de rareza, representatividad y diversidad, elaborados en el marco del proyecto FIREGLOBE sobre el mismo ámbito geográfico: España peninsular. El objetivo es conocer qué proporción de los usos del suelo más raros y representativos y qué porcentaje de los paisajes biogeográficos más diversos de este territorio se encuentran incluidos en esta red ampliada de Áreas Protegidas. Martínez-Vega et al., (2012) calcularon estos mapas empleando estos 3 indicadores de Ecología del Paisaje (índices de rareza, representatividad y diversidad de Simpson) en el entorno del programa FRAGSTATS.

Como puede apreciarse en la tabla 3, el 82,85\% de los ecosistemas forestales de España peninsular que tienen un índice de rareza superior a $0,86^{5}$ se encuentran dentro de la red ampliada de Áreas Protegidas propuesta.

Tabla 3. Superficie de los ecosistemas forestales de mayor rareza, representatividad y diversidad.

\begin{tabular}{|l|c|c|}
\cline { 2 - 3 } \multicolumn{1}{l|}{} & $\mathrm{Km}^{2}$ & $\%$ \\
\hline Ecosistemas forestales de rareza alta & 121.301 & \\
\hline Ecosistemas forestales de rareza alta en red ampliada & 99.775 & 82,25 \\
\hline Ecosistemas forestales de representatividad alta & 72.999 & \\
\hline Ecosistemas forestales de representatividad alta en red ampliada & 60.504 & 82,88 \\
\hline Ecosistemas forestales de diversidad alta & 46.643 & \\
\hline Ecosistemas forestales de diversidad alta en red ampliada & 41.608 & 89,21 \\
\hline
\end{tabular}

Fuente: SIG. Elaboración propia.

En la figura 2 se aprecia la distribución espacial de los ecosistemas forestales más raros de la península española, siguiendo las principales elevaciones montañosas y algunos de los ecosistemas esteparios del interior y otros litorales.

Adicionalmente, en la red ampliada se incluyen el $82,88 \%$ de algunos ecosistemas forestales que poseen una alta representatividad en el conjunto geográfico de Europa. Uno de los ejemplos más significativos de esta elevada representatividad es, junto a los montados portugueses, la inclusión de los típicos ecosistemas agrosilvopastorales de las dehesas españolas.

Por último, se incluyen también en esta red ampliada el 89,21\% de los ecosistemas forestales que poseen mayor diversidad paisajística (figura 3). La mayor parte de ellos se localizan en los sistemas montañosos, formando paisajes más diversos y fragmentados desde el punto de vista de la ocupación del suelo.

\footnotetext{
5 Se ha considerado el intervalo de mayor rareza. El rango de valores del índice de rareza se mueve entre $0=$ menor rareza y $1=$ máxima rareza.
} 


\section{CONCLUSIONES, DISCUSIÓN Y DESARROLLOS FUTUROS}

Desde el punto de vista metodológico, una vez más, se ha demostrado el potencial de las tecnologías de información geográfica (TIG) para la delimitación de corredores biológicos. También lo han demostrado trabajos anteriores (Walter y Craighead, 1997; Nandy et al., 2007; Vogt et al., 2007; Gurrutxaga et al., 2010; Tormos et al., 2011; Martínez-Vega y Echavarría, 2012; Clerici y Vogt, 2013), al igual que han comprobado la utilidad de aquéllas para la evaluación del estado de conservación de los corredores y para su seguimiento temporal. Se trata de una propuesta metodológica sencilla con objeto de estudiar, ahora, su viabilidad en términos políticos, funcionales y económicos.

Figura 2. Mapa de los ecosistemas forestales de mayor rareza incluidos en la red ampliada de Áreas Protegidas.

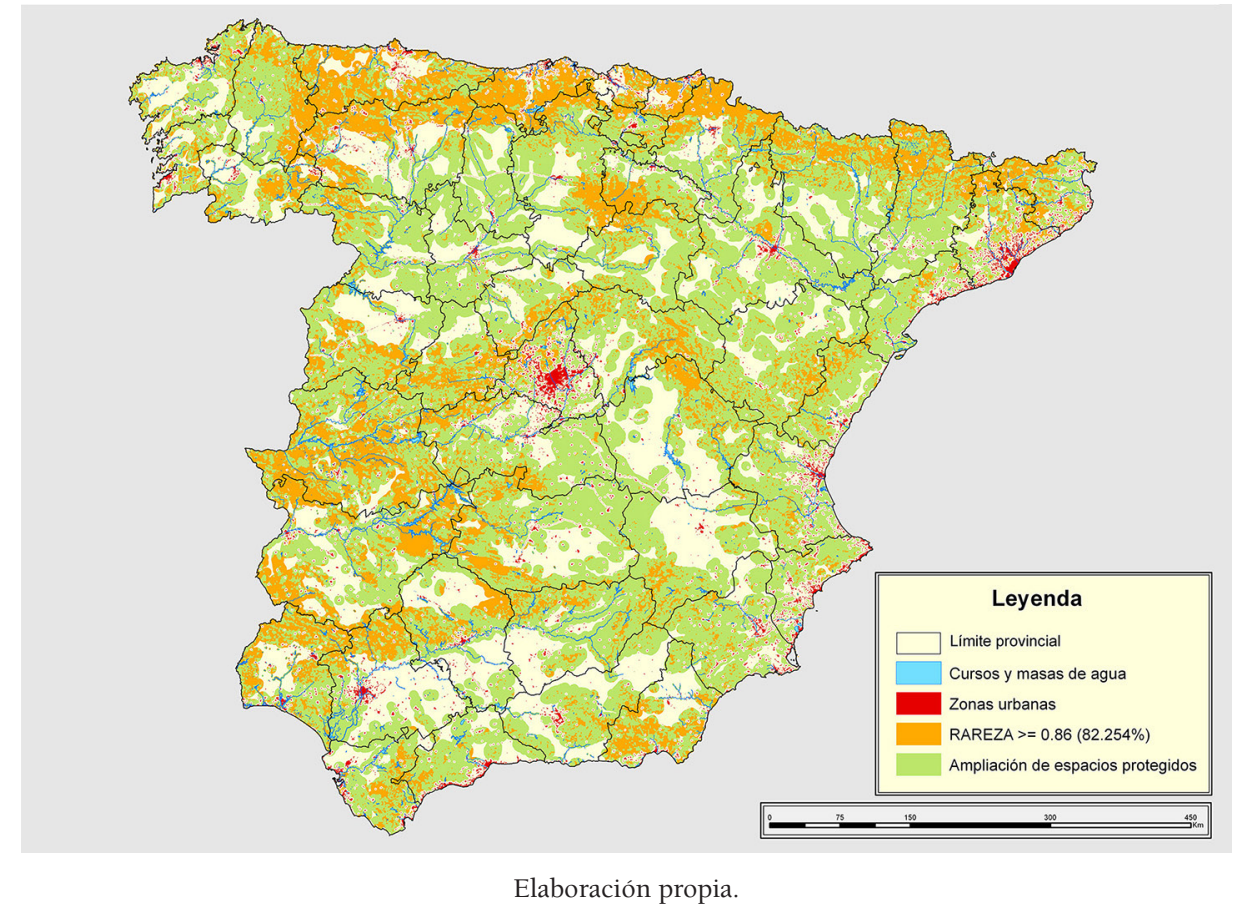

Quedan unos cuantos retos por afrontar. Desde el punto de vista operativo, será difícil de afrontar la eventual implantación de una red de este tipo. Sería necesario que algún organismo de la administración estatal (DG de Medio Natural) coordinase a todas las Comunidades Autónomas y armonizase sus prioridades e intereses con objeto de asegurar una red estatal de Áreas Protegidas que tenga coherencia y continuidad espacial.

Por otra parte, un incremento tan grande de la red de Áreas Protegidas puede ser controvertido. Científicamente, tal vez esta red ampliada proporcione una respuesta positiva a las dudas que plantean algunos investigadores acerca de si la declaración de áreas protegidas en la Península Ibérica garantiza la conservación de los recursos naturales con el fin de diseñar mejor los futuros espacios protegidos. En este sentido, Araújo et al., (2007) concluyen que, en la red actual, hay carencias. Para garantizar la conservación de determinados grupos de anfibios, reptiles, aves y flora, sería preciso incrementar el número de Áreas Protegidas, prestando especial atención a los espacios de la Red Natura 2000. La cuestión radica en saber si esta red ampliada cubriría adecuadamente estas carencias.

En el terreno operativo y financiero, esta iniciativa de red ampliada de Áreas Protegidas tiene difícil encaje en el actual Marco de Acción Prioritaria del Ministerio de Agricultura, Alimentación y Medio Ambiente y de la UE. El artículo 8 de la Directiva Hábitats establece las medidas de gestión y la disponibilidad de financiación. El reto al que se enfrentan los estados miembros es la falta de recursos económicos suficientes para gestionar los espacios actuales de la Red Natura 2000. Especialmente relevante es este asunto para España dado el gran número de espacios que aporta a la red y la gran superficie que tiene que gestionar. 
Figura 3. Mapa de los ecosistemas forestales de mayor diversidad paisajística incluidos en la red ampliada de Áreas Protegidas.

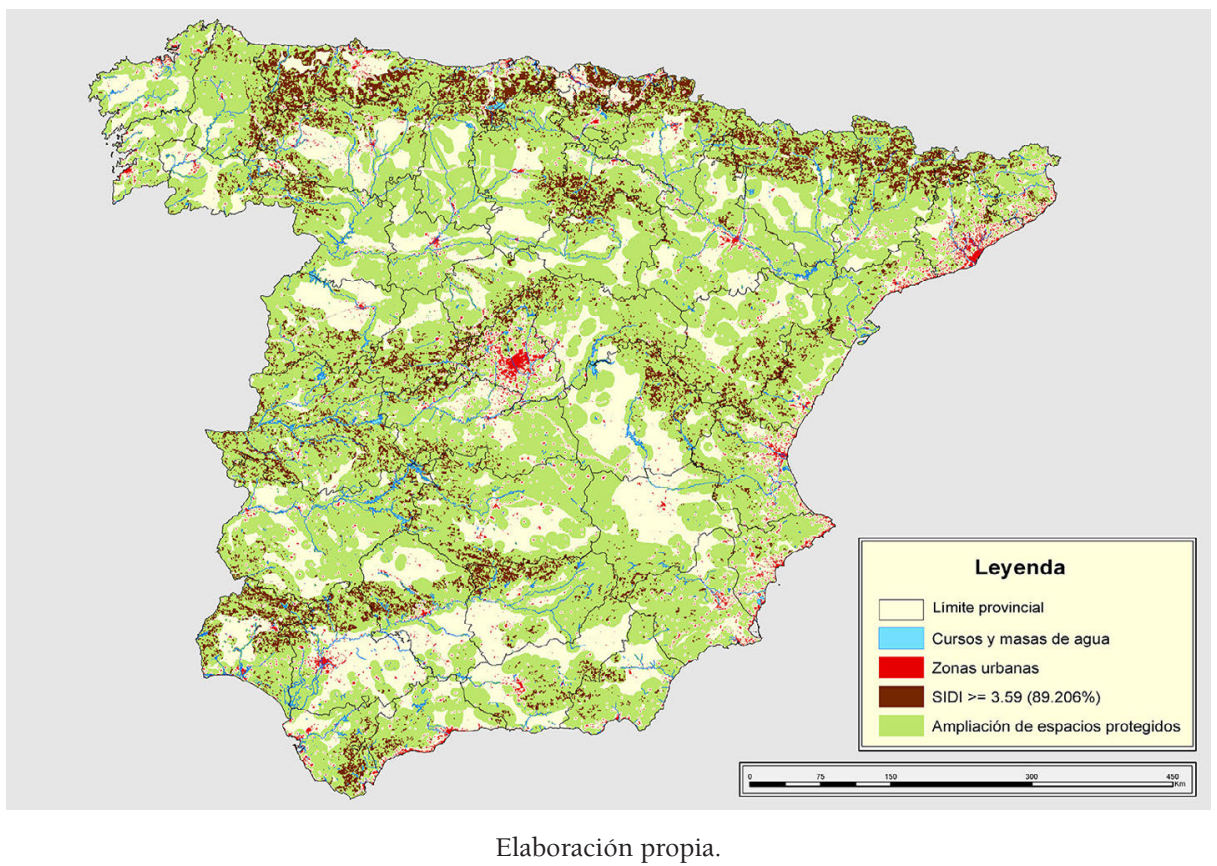

Algunas estimaciones realizadas en 2007 indicaban un coste de conservación de todos los espacios de la Red Natura 2000 cercanos a 5.000 millones de €/año. Se calcula que, en España, el coste de conservación ronda los 1.000 millones de $€ /$ año (Aymerich, 2013).

En caso de que no exista financiación suficiente, se plantea relajar la conservación de los espacios de la red Natura 2000 o, como alternativa, diseñar un plan para priorizar la conservación de los recursos naturales y espacios más importantes a través de lo que se llama Marco de Acción Prioritaria (MAP) en Red Natura 2000 con un horizonte temporal en el periodo 2014-2020. Cada estado miembro de la UE tiene que diseñar este marco, entendiendo que se trata de un instrumento de programación estratégica plurianual. No es un plan vinculante pero si orientador de la toma de decisiones de los gestores y de los financiadores. En el MAP español se han definido 850 medidas clave para los hábitats y especies implicados y se ha priorizado la inversión en la red.

Para paliar la insuficiencia de financiación a través de los canales habituales, además de utilizar los mecanismos financieros disponibles y los fondos LIFE+, se están buscando otros mecanismos alternativos de financiación: reforzamiento del programa LIFE-Biodiversidad, pagos por servicios ambientales, compensaciones, etc.

La Comisión de la UE ha realizado un estudio de relación costes-beneficios de la Red Natura 2000 y ha estimado que, aunque los costes de conservación de los espacios de la red se sitúan en torno a los 5.000 millones de €/año, los beneficios podrían estimarse entre 200.000 y 300.000 millones de $€ /$ año.

Bajo un escenario político-económico de este tipo, podría ser aceptable y viable una propuesta similar a la que se plantea en este trabajo: la ampliación de una red de Áreas Protegidas en España.

Otro aspecto discutido es la anchura de las zonas de amortiguación y de los corredores biológicos. Dependiendo de los recursos a proteger -flora y/o fauna y, en concreto, de las características y hábitos de las especies animales que se pretenden proteger-, existe una amplia variedad de anchuras utilizadas en trabajos previos sobre corredores biológicos: de 20 a $100 \mathrm{~m}$. (Bullock y Samways, 2005), de 30 a $40 \mathrm{~m}$. e incluso a $200 \mathrm{~m}$. (Laurance y Laurance, 1999), de 20 a $60 \mathrm{~m}$. (Tubelis et al., 2004), $375 \mathrm{~m}$. (Roy y Georges, 2007) y, en algunos casos, se trabaja con una anchura variable, en función de criterios definidos previamente, en torno a los $2.000 \mathrm{~m}$. (Gurrutxaga, 2008). Así pues, éste es un tema sobre el que es preciso seguir trabajando para estudiar los efectos biológicos y socioeconómicos de considerar distintos tamaños de amplitud de los corredores. 
En posteriores trabajos se pretende calcular un índice de conectividad, a nivel de paisaje, definido por el número de uniones funcionales entre todas las manchas del mismo tipo de uso del suelo (McGarigal et al., 2002, 2007), considerando los grandes usos del suelo (artificial, agrícola, forestal, otros). A la inversa, se calculará un índice de fragmentación, a nivel de paisaje, considerando una ventana móvil de 1 $\mathrm{Km}^{2}$ sobre el mapa de usos, reclasificado en las tres categorías antes mencionadas. Estos índices podrían calcularse de forma dinámica en el tiempo, considerando los mapas de CORINE-Land Cover de 2006 y 2012 y compararlos con los escenarios tendenciales y verdes que podrían construirse para algún año futuro de referencia (2025) con objeto de ayudar en el proceso de toma de decisiones.

En resumen, es preciso seguir trabajando en estas líneas para hacer una propuesta definitiva de un sistema completo de Áreas Protegidas.

\section{AGRADECIMIENTOS}

Se agradece al Ministerio de Ciencia e Innovación y al $7^{\circ}$ Programa Marco de la UE la financiación de los proyectos FIREGLOBE y SUSTAINMED, que han servido de base al trabajo desarrollado en este artículo.

\section{BIBLIOGRAFÍA}

ARAÚJO, M.B., LOBO, J. M. y MORENO, J.C. (2007): "The effectiveness of Iberian Protected Areas in conserving terrestrial biodiversity" Conservation Biology, vol. 21, nº 6, pp. 1423-1432.

ARENDT, R. (2004): "Linked landscapes. Creating greenway corridors through conservation subdivision design strategies in the northeastern and central United States", Landscape and Urban Planning, $\mathrm{n}^{\circ} 68$, pp. 241-269.

AYMERICH, M. (2013): Marco de Acción Prioritaria en Red Natura 2000, CSIC-Fundación Biodiversidad, Madrid, Comunicación personal.

BENNETT, G. y MULONGOY, K. J. (2006): Review of experience with ecological networks, corridors and buffer zones, Secretariat of the Convention on Biological Diversity, Montreal, Technical Series no 23, 100 p.

BOSQUE, J. y VILÀ, J. (1989): Geografía de España, Planeta, Madrid.

BROWN, R. y HARRIS, G. (2005): "Comanagement of wildlife corridors: the case for citizen participation in the Algonquin to Adirondack proposal", Journal of Environmental Management, n 74, pp. 97-106.

BULLOCK, W. y SAMWAYS, M. (2005): "Conservation of flower-arthropod associations in remnant African grassland corridors in an afforested pine mosaic", Biodiversity and Conservation, n 14, pp. 3093-3103.

CHUVIECO, E., AGUAdO, I., JURDAO, S., PETTINARI, M. L., YEBRA, M., SAlAS, J., HANTSON, S., DE LA RIVA, J., IBARRA, P., RODRIGUES, M., ECHEVERRÍA, M., AZQUETA, D., ROMÁN, M. V., BASTARRIKA, A., MARTÍNEZ, S., RECONDO, C., ZAPICO, E. y MARTÍNEZ-VEGA, J. (2012): "Integrating geospatial information into fire risk assessment", International Journal of Wildland Fire, http://www.publish.csiro.au/paper/WF12052.htm

CLERICI, N. y VOGT, P. (2013): "Ranking European regions as providers of structural riparian corridors for conservation and management purposes", International Journal of Applied Earth Observation and Geoinformation, $\mathrm{n}^{\circ} 21$, pp. 477-483.

DE LUCIO, J. V., MÚGICA, M., GÓMEZ-LIMÓN, J., MARTÍNEZ, C., PUERTAS, J. y ATAURI, J. A. (2008): Anuario Europarc-España del estado de los espacios naturales protegidos 2007, Fundación Fernando González Bernáldez, Madrid, 224 p. Disponible en: http://www.redeuroparc.org/documentos anexos/ Publicaciones/Anuario/anuario2007.pdf

EUROPARC-ESPAÑA:

- (2002): Plan de acción para los Espacios Naturales Protegidos del estado español, Fundación Fernando González Bernáldez, Madrid, 168 pp.

- (2005a): Integración de los espacios naturales protegidos en la ordenación del territorio, Fundación Fernando González Bernáldez, Madrid, 120 p. 
- (2005b): Procedimiento de asignación de las categorías de manejo UICN a los espacios naturales protegidos, Oficina Técnica de EUROPARC-España, http://www.sgea.org/documentos/000033 categorias uicn.pdf

- (2008): "ESPARC 2008: Avances en la gestión eficaz de las áreas protegidas: retos hasta el 2013", Actas del XIV Congreso de Europarc-España. Madrid, Fundación Fernando González Bernáldez, 144 p.

- (2012): Anuario 2011 del estado de las Áreas Protegidas en España, FUNGOBE, Madrid, 186 pp.

FAO (1976): A Framework for Land Evaluation. Soil Bulletin. 32. Rome.

GALlARDO, M. y MARTínEZ-VEGA, J. (2012): "Cambios de usos del suelo en la Comunidad de Madrid: analizando el pasado y simulando el futuro", En: Martínez-Vega, J. y Martín Isabel, M.P. (Eds.), Tecnologías de la Información Geográfica en el contexto del Cambio Global, CSIC-AGE, Madrid, pp. 305-314.

GRAVES, T.A., FARLEY, S., GOLDSTEIN, M. I. y SERVHEEN, C. (2007): "Identification of functional corridors with movement characteristics of brown bears on the Kenai Peninsula, Alaska", Landscape Ecology, no 22, pp. 765-772.

GURRUTXAGA, M. (2008): "Patrones de cobertura y protección de los bosques naturales en el País Vasco", Geographicalia, n 53, pp. 49-72.

GURRUTXGA, M., LOZANO, P. J. y DEL BARRIO, G. (2010): "GIS-based approach for incorporating the connectivity of ecological networks into regional planning", Journal for Nature Conservation, $\mathrm{n}^{\circ} 18$, pp. 318-326.

GURRUTXAGA, M., RUBIO, L. y SAURA, S. (2011): "Key connectors in protected area networks and the impact of highways: A transnational case study from the Cantabrian Range to the Western Alps (SW Europe)", Landscape and Urban Planning, n 101, pp. 310-320.

HILTY, J. A., LIDICKER, Jr., WILLIAM, Z. y MERENLENDER, A. M. (2006): Corridor Ecology: The science and practice of linking landscapes for Biodiversity Conservation, Island Press, 324 pp.

JEPSEN, J. U., BAVECO, J. M., TOPPING, C. J., VERBOOM, J. y VOS, C. C. (2005): "Evaluating the effect of corridors and landscape heterogeneity on dispersal probability: a comparison of three spatially explicit modelling approaches", Ecological Modelling, n 181, pp. 445-459.

JORDÁN, F., BÁLDI, A., ORCI, K.M., RÁCZ, I. y VARGA, Z. (2003): "Characterizing the importance of habitat patches and corridors in maintaining the landscape connectivity of a Pholidoptera transsylvanica (Orthoptera) metapopulation", Landscape Ecology, n 18, pp. 83-92.

KALIGARIC, M., SEDONJA, J. y SAJNA, N. (2008): "Traditional agricultural landscape in Goricko Landscape Park (Slovenia): distribution and variety of riparian stream corridors and patches", Landscape and Urban Planning, $\mathrm{n}^{\circ} 85$, pp. 71-78.

LAURANCE, S. G. y LAURANCE, W. F. (1999): "Tropical wildlife corridors: use of linear rainforest remnants by arboreal mammals", Biological Conservation, no 91 (2-3), pp. 231-239.

LEIBENATH, M., BLUM, A. y STUTZRIEMER, S. (2010): “Transboundary cooperation in establishing ecological networks: The case of Germany's external borders", Landscape and Urban Planning, $\mathrm{n}^{\circ} 94$, pp. 84-93.

MARTÍN, B., ORTEGA, E., MANCEBO, S. y OTERO, I. (2008): "Fragmentación de los hábitats de la Red Natura 2000 afectados por el PEI (Plan Estratégico de Infraestructuras y Transporte", Geofocus, n 8, pp. $44-60$.

MARTÍNEZ VEGA, J., MARTÍN ISABEL, M. P. y ROMERO CALCERRADA, R. (2003): "Valoración del paisaje en la Zona de Especial Protección de Aves Carrizales y Sotos de Aranjuez (Comunidad de Madrid)", Geofocus, no 3, pp. 1-21.

MARTÍNEZ-VEGA, J. y CHUVIECO, E. (2012): "Elaboración de un índice sintético de riesgo de incendios forestales en España Peninsular", Boletín de la Real Sociedad Geográfica, no CXLVIII, pp. $73-94$. 
MARTÍNEZ VEGA, J. y ECHAVARRÍA DASPET, P. (2012): "Hacia una estrategia global de conservación de la naturaleza en la provincia de Cuenca", Boletín de la Asociación de Geógrafos Españoles, $\mathrm{n}^{\circ}$ 59, pp. 201-220.

MARTÍNEZ-VEGA, J., ECHAVARRÍA, P., IBARRA, P., ECHEVERRÍA, M. y RODRIGUES, M. (2012): "Valoración del paisaje de España peninsular en el contexto de la generación de un índice sintético de riesgo de incendios forestales", En: Martínez-Vega, J. y Martín Isabel, M. P. (Eds.), Tecnologías de la Información Geográfica en el contexto del Cambio Global, CSIC-AGE, Madrid, pp. 133-142.

McGARIGAL, K., MARKS, B., HOLMES, C. y ENE, E. (2002): Fragstats 3.3. Spatial Pattern Analysis Program for Quantifying Landscape Structure, Department of Natural Resources Conservation, University of Massachusetts.

McGARIGAL, K., ENE, E. y HOLMES, C. (2007): Spatial Pattern Analysis Program for Categorical Maps, University of Massachusetts. http://www.umass.edu/landeco/research/fragstats/fragstats.html

MINISTERIO DE MEDIO AMBIENTE (1999): Estrategia española para la conservación y el uso sostenible de la diversidad biológica, Ministerio de Medio Ambiente, Madrid, 160 pp.

MINISTERIO DE PRESIDENCIA (2007): Estrategia española de Desarrollo Sostenible, Ministerio de Presidencia, Madrid, 122 pp. Disponible en: http://www.la-moncloa.es/NR/rdonlyres/B73920C0-8F78-4EFE-83D8-A570345ADBA4/0/EEDS.pdf.

NANDY, S., KUSHWAHA, S. P. S., MUKHOPADHYAY, S. (2007): "Monitoring the Chilla-Motichur wildlife corridor using geospatial tools", Journal for Nature Conservation, $n^{\circ}$ 15, pp. 237-244.

OSE (2012): Sostenibilidad en España 2012, Observatorio de la Sostenibilidad en España, Alcalá de Henares.

PARKER, K., HEAD, L., CHISHOLM, L.A. y FENELEY, N., (2008): "A conceptual model of ecological connectivity in the Shellharbour Local Government Area, New South Wales, Australia", Landscape and Urban Planning, $\mathrm{n}^{\circ}$ 86, pp. 47-59.

RODRÍGUEZ-RODRÍGUEZ, D. (2012): "Perception, use and valuation of Protected Areas in an economic crisis context", Environmental Conservation, no 39, pp. 162-171.

RODRÍGUEZ-RODRÍGUEZ, D. y MARTÍNEZ-VEGA, J.:

- (2012): "Proposal of a system for the integrated and comparative assessment of protected areas", Ecological Indicators, ${ }^{\circ}$ 23, pp. 566-572. Appendix A: Supplementary data. http://dx.doi. org/10.1016/j.ecolind.2012.05.009

- (2013a): Evaluación de la eficacia de las áreas protegidas. El Sistema de Evaluación Integrada de Áreas Protegidas (SEIAP). Resultados de la primera evaluación integrada de los espacios naturales protegidos de la Comunidad de Madrid, Fundación BBVA, Bilbao, 314 pp., https://digital.csic.es/handle/10261/76701

- (2013b): "Results of the implementation of the System for the Integrated Assessment of Protected Areas (SIAPA) to the protected areas of the Autonomous Region of Madrid (Spain)", Ecological Indicators, ${ }^{\circ} 34$, pp. 210-220.

http://www.sciencedirect.com/science/article/pii/S1470160X13001829

ROE, J. H. y GEORGES, A. (2007): "Heterogeneous wetland complexes, buffer zones, and travel corridors: Landscape management for freshwater reptiles", Biological Conservation, $\mathrm{n}^{\circ}$ 135, pp. 67-76.

ROSA, D. de la, MAYOL, F, DIAZ-PEREIRA, E., FERNÁNDEZ, M. y ROSA Jr., D. de la (2004): "A land evaluation decision support system (MicroLEIS DSS) for agricultural soil protection with special reference to the Mediterranean region", Environmental Modelling \& Software, n 19, pp. 929-942.

ROSA, D. de la, ANAYA-ROMERO, M., DIAZ-PEREIRA, E., HEREDIA, N. y SHAHBAZI, F. (2009): "Soil-specific agro-ecological strategies for sustainable land use. A case study by using MicroLEIS DSS in Sevilla Province (Spain)", Land Use Policy, n 26, pp. 1055- 1065.

ROY, V. y BLOIS, S. (2008): "Evaluating hedgerow corridors for the conservation of native forest herb diversity", Biological Conservation, $\mathrm{n}^{\circ} 141$, pp. 298-307. 
SILORI, C. S. y MISHRA, B. K. (2001): "Assessment of livestock grazing pressure in and around the elephant corridors in Mudumalai Wildlife Sanctuary, south India”, Biodiversity and Conservation, $\mathrm{n}^{\circ} 10$, pp. 2181-2195.

SUSKEVICS, M., TILLEMANN, K. y KÜLVIK, M. (2013): "Assessing the relevance of stakeholder analysis for national ecological network governance: The case of the Green Network in Estonia", Journal for Nature Conservation, $\mathrm{n}^{\circ}$ 21, pp. 206-213.

TORMOS, T., KOSUTH, P., DURRIEU, S., VILLENEUVE, B. y WASSON, J.G. (2011): "Improving the quantification of land cover pressure on stream ecological status at the riparian scale using High Spatial Resolution Imagery", Physics and Chemistry of the Earth, $\mathrm{n}^{\circ}$ 36, pp. 549-559.

TUBELIS, D.P., COWLING, A. y DONNELlY, C. (2004): "Landscape supplementation in adjacent savannas and its implications for the design of corridors for forest birds in the central Cerrado, Brazil", Biological Conservation, $\mathrm{n}^{\circ} 118$, pp. 353-364.

VOGT, P., RIITERS, K.H., IWANOWSKI, M., ESTREGUIL, C., KOZAK, J. y SOILLE, P. (2007): "Mapping landscape corridors", Ecological Indicators, $\mathrm{n}^{\circ}$ 7, pp. 481-488.

WALKER, R. y CRAIGHEAD, L. (1997): Analyzing wildlife movement corridors in Montana using GIS, disponible en: http://proceedings.esri.com/library/userconf/proc97/proc97/to150/pap116/papl16.htm

WEBER, T., SLOAN, A. y WOLF, J. (2006): "Maryland's Green Infrastructure assessment: development of a comprehensive approach to land conservation", Landscape and Urban Planning, $\mathrm{n}^{\circ}$ 77, pp. 94-110.

WISSMAR, R.C. (2004): "Riparian corridors of Eastern Oregon and Washington: functions and sustainability along lowland-arid to mountains gradients", Aquatic Sciencies, nº 66, pp. 373-387.

WOESS, M., GRILLMAYER, R. y VOELK, F. H. (2002): "Green bridges and wildlife corridors in Austria", Zeitschrift für Jagdwissenschaft, $\mathrm{n}^{\circ} 48$, pp. 25-32. 